\title{
Fujita modified exponent for scale invariant damped semilinear wave equations
}

\author{
Felisia Angela Chiarello (id, Giovanni Girardi and SAndra Lucente
}

Abstract. The aim of this paper is to prove a blow-up result of the solution for a semilinear scale invariant damped wave equation under a suitable decay condition on radial initial data. The admissible range for the power of the nonlinear term depends both on the damping coefficient and on the pointwise decay order of the initial data. In addition, we give an upper bound estimate for the lifespan of the solution. It depends not only on the exponent of the nonlinear term and not only on the damping coefficient but also on the size of the decay rate of the initial data.

\section{Introduction}

In the recent years, the following Cauchy problem for the wave equation with scale invariant damping spreads a new line of research on variable coefficient-type equations. More precisely, we are dealing with

$$
\left\{\begin{array}{l}
v_{t t}(t, x)-\Delta v(t, x)+\frac{\mu}{1+t} v_{t}(t, x)+\frac{v}{(1+t)^{2}} v(t, x)=|v(t, x)|^{p}, \quad t \geq 0, \quad x \in \mathbb{R}^{n}, \\
v(0, x)=0 \\
v_{t}(0, x)=\varepsilon g(x),
\end{array}\right.
$$

with $n \geq 2, \mu, v \in \mathbb{R}, p>1$ and $g$ a radial smooth function. In [2,3,10,11], some results on the global existence of a solution for (1) with non-compactly initial data appeared assuming a suitable decay behavior for $g$. Many other results concern blowup and global existence for this equation, see [12] for a summary of this problem. The main point is to find a critical exponent, fixed a suitable space of data. More precisely, a level $\bar{p}$ is critical if for $p>\bar{p}$, one can prove that for $\epsilon>0$ sufficiently small and for any $g$ chosen in the fixed space, there exists a unique global (in time) solution of the problem, and conversely, if $p \in(1, \bar{p})$, for any $\epsilon>0$ there exist some $g$ in this space such that the local solution cannot be prolonged over a finite time. Coming back to (1), in dependence on $\mu, v$ and $n$, a competition between two critical exponents appeared. In some cases, the Strauss exponent is dominant; it is given by the wave

Mathematics Subject Classification: Primary 35B33, Secondary 35L70

Felisia Angela Chiarello: Supported by "Compagnia di San Paolo" (Torino, Italy). Sandra Lucente: Partially supported by PRIN 2017- linea Sud "Qualitative and quantitative aspects of nonlinear PDEs." 
equation theory, it will be denoted by $p_{S}(d)$, and it is the positive root of the quadratic equation

$$
(d-1) p^{2}-(d+1) p-2=0 .
$$

For other assumptions, the equation goes to an heat equation and a Fujita-type exponent $p_{F}(h):=1+\frac{2}{h}$ appears. In all known results, the quantities $d>1, h>0$ depend on $v, \mu$ and $n$. Changing the space of data, a change of critical exponent may appear. The novelty of our result consists in showing that if one takes into account the decay rate of the initial data, then the Fujita-type exponent depends also on such decay rate. In addition, we give an upper bound estimate for the lifespan of the solution, in terms of the power of the nonlinear term, the size and the growth of the initial data. Let us recall that the lifespan of the solution is a function of $\varepsilon$ which gives the maximal existence time:

$T(\varepsilon):=\sup \left\{T>0\right.$ such that the local solution $u$ to (1) is defined on $\left.[0, T) \times \mathbb{R}^{n}\right\}$.

Finally, we will prove the following.

Theorem 1. Let $n \geq 2$. Let $\epsilon>0$ and $g$ be a radial smooth function satisfying

$$
g(|x|) \geq \frac{M}{(1+|x|)^{\bar{k}+1}} \text { with } \bar{k}>-1,
$$

for some $M>0$ and for any $x \in \mathbb{R}^{n}$. Assuming in addition that

$$
\bar{k}+\frac{\mu}{2}>0, \quad \frac{\mu}{2}\left(\frac{\mu}{2}-1\right) \geq v
$$

and

$$
1<p<p_{F}\left(\bar{k}+\frac{\mu}{2}\right)
$$

then the classical solution of (1) blows up. More precisely, the lifespan of the solution $T(\varepsilon)>0$ is finite and satisfies

$$
T(\varepsilon) \leq C \epsilon^{-\frac{2(p-1)}{4-(\mu+2 \tilde{k})(p-1)}},
$$

with $C>0$, independent of $\varepsilon$.

Remark 1. Recently, Ikeda, Tanaka, Wakasa in [6] consider a similar question for cubic convolution nonlinearity and a critical decay appears.

Remark 2. In [4], we will also consider a variant of problem (1), in which the nonlinearity depends on $v, t, v_{t}$ combined in a suitable way.

Remark 3. The lifespan estimate for the same equation with compactly supported data and $v \neq \frac{\mu}{2}\left(\frac{\mu}{2}-1\right)$ has been considered in [13]. If $v \leq \frac{\mu}{2}\left(\frac{\mu}{2}-1\right)$, the lifespan estimate is different from (3) due to the compactness of the support of the initial data.

The paper is organized as follows: In Sect. 2, we give an overview of the known results and we state an auxiliary theorem; in Sect. 3, we prove the main results. 


\section{Motivations}

\subsection{The case $\mu=2, v=0$}

Let us start with a quite simple case

$$
\left\{\begin{array}{l}
v_{t t}-\Delta v+\frac{2}{1+t} v_{t}=|v|^{p}, \quad t \geq 0, \quad x \in \mathbb{R}^{n}, \\
v(0, x)=0, \\
v_{t}(0, x)=\operatorname{gg}(x) .
\end{array}\right.
$$

The global existence of small data solutions for this problem was first solved in [1] for a suitable range of $n$ and $p$. Some nonexistence results were also established for $p<p_{F}(n):=1+\frac{2}{n}$. Except for the one-dimensional case, a gap between this value and the admissible exponents in [1] appeared. In [3] for dimension $n=2,3$, this gap was covered with an unexpected result. Indeed, in that paper the Strauss exponent came into play. Afterward, the global existence of small data solutions to (4) has been proved for any $p>p_{S}(n+2)$ also in odd dimension $n \geq 5$ in [2] and in even dimension $n \geq 4$ in [10].

Moreover, we know that the exponent $p_{2}(n):=\max \left\{p_{S}(n+2), p_{F}(n)\right\}$ is optimal; in fact, in [3], the authors prove the blowup of solutions of (4) for each $1<p \leq p_{2}(n)$ in each dimension $n \in \mathbb{N}$. In $[2,3,10]$, the authors prove a global existence result not necessarily when the initial datum $g=g(x)$ has compact support. More precisely, let $n \geq 3$, given a radial initial datum $g(x)=g(|x|)$ with $g \in C^{1}(\mathbb{R})$, for any $p>p_{S}(n+2)$ it is possible to choose $\bar{k}>0$ and $\epsilon_{0}>0$ such that (4) admits a radial global solution $u \in C\left([0, \infty) \times \mathbb{R}^{n}\right) \cap C^{2}\left([0, \infty) \times \mathbb{R}^{n} \backslash\{0\}\right)$ provided

$$
\varepsilon \leq \varepsilon 0, \quad\left|g^{(h)}(r)\right| \leq \epsilon\langle r\rangle^{-(\bar{k}+1+h)} \text { for } h=0,1 .
$$

In the present paper, we discuss the dependence of $\bar{k}$ from $n$ and $p$. In (5), the exponent $\bar{k}$ has to belong to a suitable interval $\left[k_{1}(n, p), k_{2}(n, p)\right]$. It is interesting to investigate the case of $\bar{k} \notin\left[k_{1}(n, p), k_{2}(n, p)\right]$. In the sequel, we will see that the bound $k_{2}(n, p)$ can be easily improved (see Remark 4). On the contrary, if $k<k_{1}(n, p)$, then a new result appears. The known situation is the following:

$$
\begin{aligned}
- & k_{1}(3, p)=\max \left\{\frac{3-p}{p-1}, \frac{1}{p-1}\right\} \text { and } k_{2}(3, p)=2(p-1) \text {, see [3]. } \\
- & k_{1}(n, p)=\max \left\{\frac{3-p}{p-1}, \frac{n-1}{2}\right\} \text { and } k_{2}(n, p)=\min \left\{\frac{(n+1) p}{2}-2, \frac{n^{2}-2 n+13}{2(n-3)}\right\} \text { if } \\
& n \geq 5 \text { odd, see }[2] . \\
- & k_{1}(n, p)=\max \left\{\frac{3-p}{p-1}, \frac{n-1}{2}\right\} \text { and } k_{2}(n, p)=\min \left\{\frac{(n+1) p}{2}-2, n-1\right\} \text { if } n \geq 4, \\
& \text { see [10]. }
\end{aligned}
$$

We can write in a different way the previous conditions. Firstly, we concentrate on the case $n=3$. For $p \in(1,2)$, we have $\bar{k} \geq \frac{3-p}{p-1}$ that is equivalent to

$$
p \geq 1+\frac{2}{\bar{k}+1}=p_{F}(\bar{k}+1) .
$$




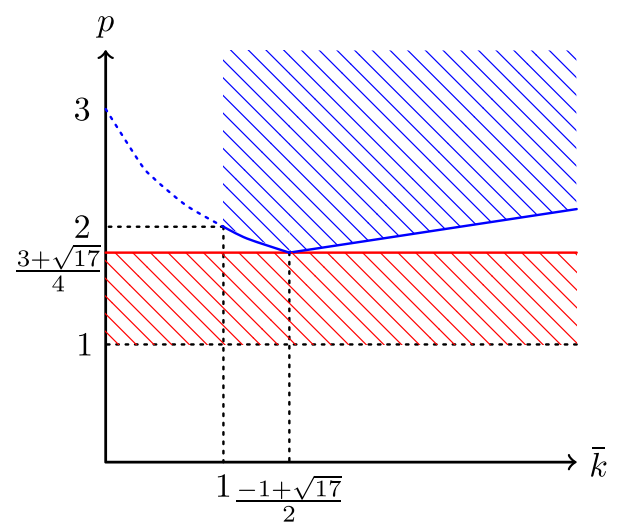

Figure 1. $n=3, \mu=2, v=0$

From above, we have $\bar{k} \leq 2(p-1)$ that is

$$
p \geq \frac{\bar{k}}{2}+1
$$

The intersection of $p=p_{F}(\bar{k}+1)$ and $p=1+\bar{k} / 2$ is exactly in $\bar{k}=\frac{-1+\sqrt{17}}{2}$ and $p=p_{S}(5)$. We summarize the situation in Fig. 1. In the following graphs, we denote in blue the zone of the known global existence results, in red the zone of the known blow-up results. In this paper, we want to cover the white zones.

Reading [2], we see that the same situation appears for any odd $n \geq 5$. The critical curve

$$
p=p_{F}(\bar{k}+1)
$$

intersects the line

$$
p=\frac{2(\bar{k}+2)}{n+1}
$$

in the Strauss couple

$$
\left(\bar{k}_{0}, \frac{2\left(\bar{k}_{0}+2\right)}{n+1}\right)=\left(\frac{n-5+\sqrt{n^{2}+14 n+17}}{4}, p_{S}(n+2)\right) .
$$

The only difference with the case $n=3$ is that, in the global existence zone, a bound from above appears for $p$ and this has some influence on $k_{2}(n, p)$. More precisely, one can take

$$
p \leq \frac{n+1}{n-3}, \quad \bar{k} \leq \frac{n^{2}-2 n+13}{2(n-3)} \quad \text { if } n \geq 7,
$$

and $p \leq 2, \bar{k} \leq 3$ if $n=5$. Hence, the result of such paper can be represented as in Fig. 2. Our aim is to prove blowup in the white zone below the Fujita curve. 


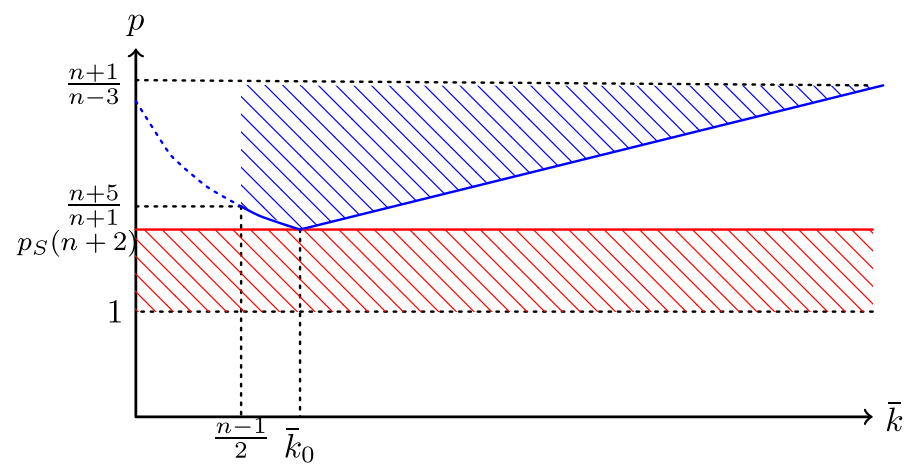

Figure 2. $n \geq 5$ odd, $\mu=2, v=0$

Even dimension is more delicate. In [10], the global existence result is established in the blue zone below the line $p=\frac{n+5}{n+1}$ except on the curve $p=p_{F}(\bar{k}+1)$. For convenience of the reader, we precise that in the notation of [10], the role of $\bar{k}$ is taken by the quantity $k+\frac{n+1}{2}$.

\subsection{The case $\mu>2$ and $v=\frac{\mu}{2}\left(\frac{\mu}{2}-1\right)$}

In $[10,11]$, the author considers the Cauchy problem (1) for the semilinear wave equation with scale invariant damping and mass terms, that is $v=\frac{\mu}{2}\left(\frac{\mu}{2}-1\right) \geq 0$. We see that for $\mu=2$, it reduces to (4). Global existence of solutions to (1) holds under the conditions

$$
\mu \in[2, M(n)], \quad M(n)=\frac{n-1}{2}\left(1+\sqrt{\frac{n+7}{n-1}}\right) .
$$

In the even case [10], the initial data satisfy (5) for $\bar{k} \in\left(k_{1}(n, p, \mu), k_{2}(n, p, \mu)\right]$ such that

$$
\begin{aligned}
& k_{1}(n, p, \mu)=\max \left\{\frac{n-1}{2}, \frac{2}{p-1}-\frac{\mu}{2}\right\} \\
& k_{2}(n, p, \mu)=\min \left\{n-1, \frac{n+\mu-1}{2} p-\frac{\mu+2}{2}\right\} .
\end{aligned}
$$

Rewriting these conditions in terms of $p$, we find that

$$
p>p_{F}\left(\bar{k}+\frac{\mu}{2}\right), \quad p \geq \frac{2 \bar{k}+\mu+2}{n+\mu-1} .
$$

The intersection of the curves those define the global existence zone gives $p=p_{S}(n+$ $\mu)$. Hence, the condition $p>p_{S}(n+\mu)$ appears. Moreover, another bound from above appears:

$$
p<\bar{p}:=\min \left\{p_{F}(\mu), p_{F}\left(\frac{n+\mu-1}{2}\right)\right\} .
$$




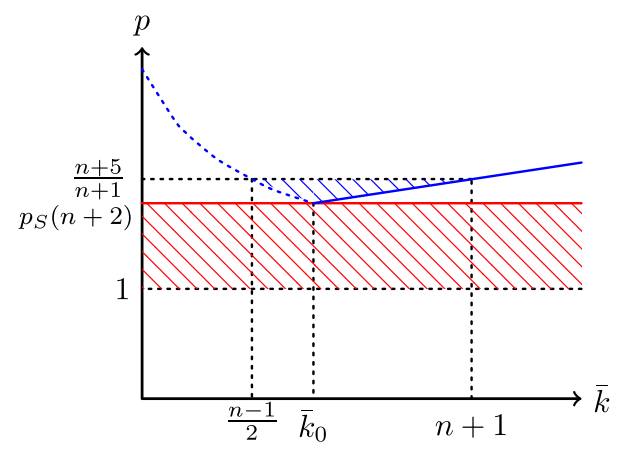

Figure 3. $n \geq 4$ even, $\mu=2, v=0$

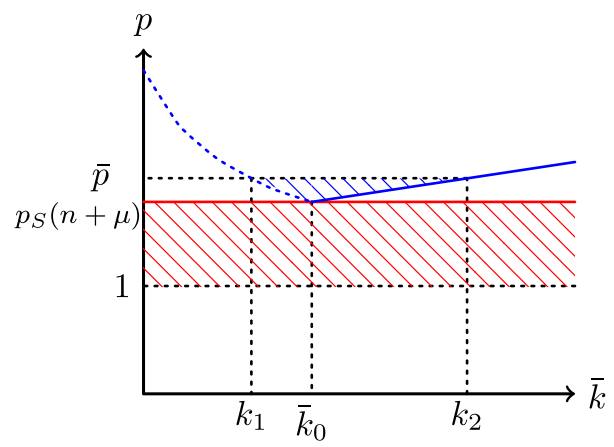

Figure 4. $n \geq 4$ even, $v=\frac{\mu}{2}\left(\frac{\mu}{2}-1\right) \geq 0$

This means that different results for large $\mu$ and small $\mu$ hold. This influences the positions of $k_{1}$ and $k_{2}$. For our purpose, it is sufficient to say that for $\mu \neq 2$ and even $n$, the situation is similar to Fig. 3. More precisely, in Fig. $4 p_{S}(n+\mu)$ appears. The blow-up result is indeed given in [9]. The zone between $p=p_{S}(n+\mu)$ and $p=p_{F}\left(\vec{k}+\frac{\mu}{2}\right)$ is not covered by any known result.

The corresponding global existence result for the Cauchy problem (1) in odd space dimension $n \geq 1$ is studied in [11] for radial and small data, assuming condition (5) with $\bar{k} \in\left[k_{1}(n, p, \mu), k_{2}(n, p, \mu)\right]$ where $k_{2}$ satisfies (7) and it holds:

$$
\begin{aligned}
& k_{1}(3, p, \mu)=\max \left\{1, \frac{2}{p-1}-\frac{\mu}{2}, \frac{1}{p-1}\right\} ; \\
& k_{1}(n, p, \mu)=\max \left\{\frac{n-1}{2}, \frac{2}{p-1}-\frac{\mu}{2}\right\}, n \geq 5 \quad \mu \in[2, n-1] ; \\
& k_{1}(n, p, \mu)=\max \left\{\frac{n-1}{2}, \frac{2}{p-1}-\frac{\mu}{2}, \frac{1}{p-1},\right\}, n \geq 5 \quad \mu \in(n-1, M(n)] .
\end{aligned}
$$

In any case, the condition $p>p_{F}\left(\bar{k}+\frac{\mu}{2}\right)$ appears. Hence, in odd space dimension $n \geq 5$ the situation is not different from Fig. 4 . 
Reading Theorem 1 in the case $v=\frac{\mu}{2}\left(\frac{\mu}{2}-1\right)$, it is clear that the aim of this paper is to find blowing-up solutions to (1) even for $p>p_{S}(n+\mu)$ by considering initial data with slow decay. More precisely, let us consider

$$
g(x) \simeq \frac{M}{(1+|x|)^{\bar{k}+1}}, \quad \text { for } \quad \frac{n-1}{2}<\bar{k}<\bar{k}_{0},
$$

where $\bar{k}_{0}$ is such that

$$
p_{F}\left(\bar{k}_{0}+\frac{\mu}{2}\right)=p_{S}(n+\mu) .
$$

We will prove the blow-up result in the left white side zones in Figs. 1, 2, 3 and 4 where $\bar{k}<\bar{k}_{0}, p>p_{S}(n+\mu)$ and $p<p_{F}\left(\bar{k}_{0}+\frac{\mu}{2}\right)$. Under the same assumption on $g$, the quoted results assure that for $p \geq p_{F}\left(\bar{k}+\frac{\mu}{2}\right)$ and $p>p_{S}(n+\mu)$, there is global existence. Hence, $p=p_{F}\left(\bar{k}+\frac{\mu}{2}\right)$ is a critical curve for the Cauchy problem (1), provided $v=\frac{\mu}{2}\left(\frac{\mu}{2}-1\right) \geq 0$.

Remark 4. Still fixing $v=\frac{v}{2}\left(\frac{v}{2}-1\right) \geq 0$, let us consider $\bar{k}>\bar{k}_{0}$ and $p>p_{S}(n+\mu)$. As discussed, the global existence results in the previous literature require $p$ above a line which depends on $\bar{k}$, because of a restriction of type $\bar{k} \leq k_{2}(n, p, \mu)$ which every time appears. Actually, this restriction can be avoided; indeed, if the initial datum satisfies (8) with $\vec{k}>k_{2}(n, p, \mu)$, then we can say that the initial datum also satisfies (5) with $\bar{k}=k_{2}(n, p, \mu)$. Hence, the global existence of a solution to (1) follows from the known results.

Remark 5. For $v=\frac{\mu}{2}\left(\frac{\mu}{2}-1\right) \geq 0$, Theorem 1 provides some new information about the solution of (1) also when $p$ belongs to the red zone of Figs. 1, 2, 3, 4 and 5. In fact, for

$$
p<\min \left\{p_{S}(n+\mu), p_{F}\left(\bar{k}+\frac{\mu}{2}\right)\right\}
$$

by the previous literature we know that the solution blows up in finite time, whereas Theorem 1 gives a lifespan estimate in the case of radial initial data with non-compact support, relating this estimate with the decay rate of the data.

2.3. The case $\mu=0$ and $v=0$

In Fig. 5, we summarize the wave equation case $\mu=v=0$. The red blow-up zone was covered by many authors, see [14] and the reference therein for the whole list of blow-up results. For $\mu=v=0$, the global existence result has been completely solved in [5], where the interested reader can find a long bibliography of previous contributes. In particular, the blue zone for radial solution without compact support assumption for the initial data has been exploited by Kubo, see, for example, [7,8]. Before these papers, Takamura obtained a blow-up result in the green zone. In [15], the point is to find a critical decay level $k_{0}=\frac{2}{p-1}$, equivalently $p \leq 1+\frac{2}{k_{0}}$. We underline that this is a Fujita-type exponent. 


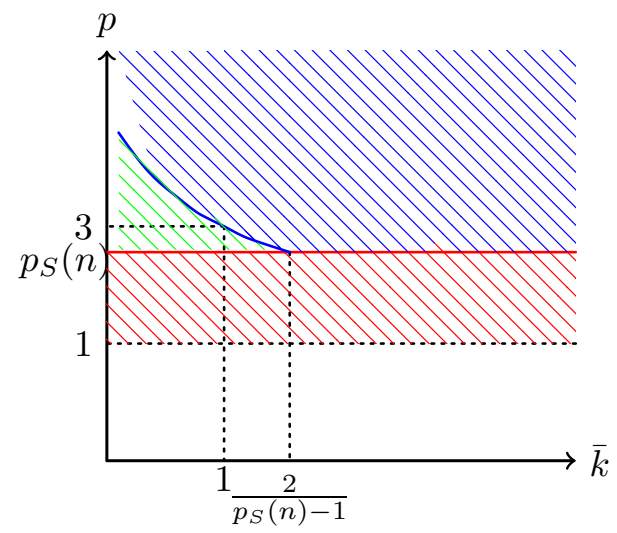

Figure 5. $\mu=v=0$

In Theorem 1, we generalize Takamura's result when $\mu \neq 0$ and $v \leq \frac{\mu}{2}\left(\frac{\mu}{2}-1\right)$. To this aim, it is sufficient to consider a peculiar wave equation with nonlinear term having a decaying time-dependent variable coefficient. This means that we will deduce Theorem 1 from the following result.

Theorem 2. Let $n \geq 2$. Given a smooth function $g=g(|x|)$ with $x \in \mathbb{R}^{n}$, we set $r=|x|$ and we consider $g=g(r)$ satisfying

$$
g(r) \geq \frac{M}{(1+r)^{\bar{k}+1}}, \text { with } \bar{k}>-1,
$$

for some $M>0$. Let $u=u(t, r)$ be the radial local solution to

$$
\left\{\begin{array}{l}
u_{t t}-u_{r r}-\frac{n-1}{r} u_{r}=(1+t)^{-\frac{\mu}{2}(p-1)}|u|^{p}, \quad r>0, \\
u(0, r)=0 \\
u_{t}(0, r)=\varepsilon g(r) .
\end{array}\right.
$$

with $p>1$ and $p<p_{F}\left(\frac{\mu}{2}-1\right)$ if $\mu>2$. Assume in addition that

$$
-1<\bar{k}<\frac{2}{p-1}-\frac{\mu}{2} \text {. }
$$

Then, given $\epsilon>0$, the lifespan $T(\varepsilon)>0$ of classical solutions to (10) satisfies

$$
T(\varepsilon) \leq C \epsilon^{-\frac{2(p-1)}{4-(\mu+2 \bar{k})(p-1)}},
$$

with $C>0$, independent of $\varepsilon$.

Remark 6. The assumption $p<p_{F}\left(\frac{\mu}{2}-1\right)$ if $\mu>2$ guarantees that the range of admissible $\bar{k}$ in (11) is not empty.

In the case $\mu=0$, Theorem 2 coincides with Takamura's result in [15]. In the proof of Theorem 2, we will follow the same approach of that paper. 


\section{Proof of the main results}

\subsection{Proof of Theorem 2}

We recall the crucial lemma of [15].

Lemma 1. Let $n \geq 2$ and $m=[n / 2]$. Given a smooth function $g=g(|x|)$ with $x \in \mathbb{R}^{n}$, we set $r=|x|$ and we consider $g=g(r)$. Let us denote by $u^{0}(t, r)$ the solution of the free wave problem

$$
\left\{\begin{array}{l}
\square u^{0}=0 \\
u^{0}(0, r)=0, u_{t}^{0}(0, r)=g(r) .
\end{array}(t, r) \in[0, \infty) \times[0, \infty)\right.
$$

Let $u=u(t, r)$ be a solution to

$$
u_{t t}-u_{r r}-\frac{n-1}{r} u_{r}=F(t, u)
$$

with the initial condition

$$
u(0, r)=0, u_{t}(0, r)=\varepsilon g(r), \quad r \in[0, \infty) .
$$

If $F$ is nonnegative, there exists a constant $\delta_{m}>0$ such that

$$
\begin{aligned}
u(t, r) & \geq \varepsilon u^{0}(t, r)+\frac{1}{8 r^{m}} \int_{0}^{t} d \tau \int_{r-t+\tau}^{r+t+\tau} \lambda^{m} F(t, u(t, \lambda)) d \lambda, \\
u^{0}(t, r) & \geq \frac{1}{8 r^{m}} \int_{r-t}^{r+t} \lambda^{m} g(\lambda) d \lambda,
\end{aligned}
$$

provided

$$
r-t \geq \frac{2}{\delta_{m}} t>0
$$

The constant $\delta_{m}$ in the previous lemma is described in [15, Lemma 2.5]; it depends on the space dimension, and in particular, it changes accordingly with the different representations of the free wave solution in odd and even dimension.

We are ready to prove that if (9) holds, then the solution of (10) blows up in finite time even for small $\varepsilon$.

Let us fix $\delta>0$; we define a blow-up set,

$$
\Sigma_{\delta}=\left\{(t, r) \in(0, \infty)^{2}: r-t \geq \max \left\{\frac{2}{\delta_{m}} t, \delta\right\}\right\},
$$

where $\delta_{m}>0$ is the constant given in Lemma 1. Combining the assumption (10) with the formulas (15) and (16), for any $(t, r) \in \Sigma_{\delta}$, it holds

$$
u(t, r) \geq \varepsilon u^{0}(t, r) \geq \frac{\varepsilon}{8 r^{m}} \int_{r-t}^{r+t} \lambda^{m} g(\lambda) d \lambda \geq \frac{M \varepsilon}{8 r^{m}} \int_{r-t}^{r+t} \lambda^{m}(1+\lambda)^{-(\bar{k}+1)} d \lambda .
$$


Then, (17) implies that

$$
\begin{aligned}
u(t, r) & \geq \frac{M \varepsilon}{8 r^{m}}\left(\frac{1+\delta}{\delta}\right)^{-(\bar{k}+1)} \int_{r-t}^{r+t} \lambda^{m-(\bar{k}+1)} d \lambda \\
& \geq \frac{M \varepsilon}{8 r^{m}}\left(\frac{1+\delta}{\delta}\right)^{-(\bar{k}+1)}(r+t)^{-(\bar{k}+1)} \int_{r-t}^{r+t} \lambda^{m} d \lambda \\
& \geq \frac{M \varepsilon}{8}\left(\frac{1+\delta}{\delta}\right)^{-(\bar{k}+1)} \frac{(r-t)^{m} 2 t}{r^{m}(r+t)^{\bar{k}+1}} .
\end{aligned}
$$

Since $(t, r) \in \Sigma_{\delta}$, we have

$$
u(t, r) \geq \frac{C_{0} t^{m+1}}{r^{m}(r+t)^{\bar{k}+1}},
$$

where we set

$$
C_{0}=\varepsilon \frac{2^{m-2} M}{\delta_{m}^{m}}\left(\frac{\delta}{1+\delta}\right)^{\bar{k}+1}>0 .
$$

Now, we assume an estimate of the form

$$
u(t, r) \geq \frac{C t^{a}}{r^{m}(r+t)^{b}} \text { for }(t, r) \in \Sigma_{\delta},
$$

where $a, b$ and $C$ are positive constant. In particular, (19) holds true for $a=m+1$, $b=\bar{k}+1$ and $C=C_{0}$.

Being $g \geq 0$, from (16) we deduce $u^{0} \geq 0$. Combining (15) and (19), for $(t, r) \in \Sigma_{\delta}$, we get

$$
\begin{aligned}
u(t, r) & \geq \frac{1}{8 r^{m}} \int_{0}^{t} d \tau \int_{r-t+\tau}^{r+t-\tau} \frac{\lambda^{m}}{(1+\tau)^{\frac{\mu}{2}(p-1)}}|u(\tau, \lambda)|^{p} d \lambda \\
& \geq \frac{C^{p}}{8 r^{m}} \int_{0}^{t} \frac{\tau^{p a}}{(1+\tau)^{\frac{\mu}{2}(p-1)}} d \tau \int_{r-t+\tau}^{r+t-\tau} \lambda^{m(1-p)}(\lambda+\tau)^{-p b} d \lambda \\
& \geq \frac{C^{p}}{8 r^{m}(r+t)^{p b+m(p-1)}} \int_{0}^{t} \frac{\tau^{p a}}{(1+\tau)^{\frac{\mu}{2}(p-1)}} d \tau \int_{r-t+\tau}^{r+t-\tau} d \lambda \\
& \geq \frac{C^{p}}{4 r^{m}(r+t)^{p b+m(p-1)}} \int_{0}^{t} \frac{(t-\tau)}{(1+\tau)^{\frac{\mu}{2}(p-1)}} \tau^{p a} d \tau
\end{aligned}
$$

By means of integration by parts, we obtain

$$
\begin{aligned}
& \int_{0}^{t} \frac{(t-\tau) \tau^{p a}}{(1+\tau)^{\frac{\mu}{2}(p-1)}} d \tau \geq \frac{1}{(1+t)^{\frac{\mu}{2}(p-1)}} \int_{0}^{t}(t-\tau) \tau^{p a} d \tau \\
& \geq \frac{1}{(1+t)^{\frac{\mu}{2}(p-1)}} \frac{t^{p a+2}}{(p a+1)(p a+2)} .
\end{aligned}
$$


While searching a finite lifespan of a solution, it is not restrictive to assume $t>1$. We have

$$
\int_{0}^{t} \frac{(t-\tau) \tau^{p a}}{(1+\tau)^{p-1}} d \tau \geq \frac{t^{p\left(a-\frac{\mu}{2}\right)+2+\frac{\mu}{2}}}{2^{p-1}(p a+1)(p a+2)} .
$$

Let $(t, r) \in \Sigma_{\delta}$, from (19)-(21), we can conclude

$$
u(t, r) \geq \frac{C^{*} t^{a^{*}}}{r^{m}(r+t)^{b^{*}}} \text { for }(t, r) \in \Sigma_{\delta}
$$

with

$$
a^{*}=p\left(a-\frac{\mu}{2}\right)+2+\frac{\mu}{2}, \quad b^{*}=p b+m(p-1), \quad C^{*}=\frac{(C / 2)^{p}}{2(p a+2)^{2}} .
$$

Let us define the sequences $\left\{a_{k}\right\},\left\{b_{k}\right\},\left\{C_{k}\right\}$ for $k \in \mathbb{N}$ by

$$
\begin{aligned}
& a_{k+1}=p\left(a_{k}-\frac{\mu}{2}\right)+2+\frac{\mu}{2}, a_{1}=m+1, \\
& b_{k+1}=p b_{k}+m(p-1), b_{1}=\bar{k}+1, \\
& C_{k+1}=\frac{\left(C_{k} / 2\right)^{p}}{2\left(p a_{k}+2\right)^{2}}, C_{1}=C_{0},
\end{aligned}
$$

where $C_{0}$ is defined by (18). Hence, we have

$$
\begin{aligned}
& a_{k+1}=p^{k}\left(m+1-\frac{\mu}{2}+\frac{2}{p-1}\right)+\frac{\mu}{2}-\frac{2}{p-1}, \\
& b_{k+1}=p^{k}(\bar{k}+1+m)-m, \\
& C_{k+1} \geq K \frac{C_{k}^{p}}{p^{2 k}}
\end{aligned}
$$

for some constant $K=K(p, \mu, m)>0$ independent of $k$. The relation (28) implies that for any $k \geq 1$, it holds

$$
\begin{aligned}
& C_{k+1} \geq \exp \left(p^{k}\left(\log \left(C_{0}\right)-S_{p}(k)\right)\right), \\
& S_{p}(k)=\Sigma_{j=0}^{k} d_{j}, \\
& d_{0}=0 \text { and } d_{j}=\frac{j \log \left(p^{2}\right)-\log K}{p^{j}} \text { for } j \geq 1 .
\end{aligned}
$$

We note that $d_{j}>0$ for sufficiently large $j$. Since $\lim _{j \rightarrow \infty} d_{j+1} / d_{j}=1 / p$, the sequence $S_{p}(k)$ converges for $p>1$ by using the ratio criterion for series with positive terms. Hence, there is a positive constant $S_{p, K} \geq S_{p}(k)$ for any $k \in \mathbb{N}$, so that

$$
C_{k+1} \geq \exp \left(p^{k}\left(\log \left(C_{0}\right)-S_{p, K}\right)\right) .
$$


Therefore, by (22), (26)-(29), we obtain

$$
u(r, t) \geq \frac{(r+t)^{m}}{r^{m} t^{-\frac{\mu}{2}+\frac{2}{p-1}}} \exp \left(p^{k} J(t, r)\right),
$$

where

$$
J(t, r):=\log \left(C_{0}\right)-S_{p, K}+\left(m+1-\frac{\mu}{2}+\frac{2}{p-1}\right) \log t-(\bar{k}+1+m) \log (r+t) .
$$

Thus, if we prove that there exists $\left(t_{0}, r_{0}\right) \in \Sigma_{\delta}$ such that $J\left(t_{0}, r_{0}\right)>0$, then we can conclude that the solution to (10) blows up in finite time, in fact

$$
u\left(t_{0}, r_{0}\right) \rightarrow \infty \text { for } k \rightarrow \infty .
$$

By the definition of $J=J(t, r)$, we find that $J(t, r)>0$ if

$$
\left(\frac{2}{p-1}-\frac{\mu}{2}-\bar{k}\right) \log t>\log \left(\frac{e^{S_{p, K}}}{C_{0}}\left(2+\frac{r-t}{t}\right)^{\bar{k}+1+m}\right) .
$$

In particular, we can take $(t, r)=\left(t, t+\max \left\{\frac{2 t}{\delta_{m}}, \delta\right\}\right) \in \Sigma_{\delta}$; then, it is enough to prove that

$$
\left(\frac{2}{p-1}-\frac{\mu}{2}-\bar{k}\right) \log t>\log \left(\frac{e^{S_{p, K}}}{C_{0}}\left(2+\frac{2}{\delta_{m}}\right)^{\bar{k}+1+m}\right) .
$$

Now, the crucial assumption (9) comes into play. The coefficient in the left side is positive, and by using (18), we find that $J(t, r)>0$ provided

$$
t>C \epsilon^{-\left(\frac{2}{p-1}-\frac{\mu}{2}-\bar{k}\right)^{-1}}
$$

where

$$
C=\left(\frac{e^{S_{p, K}} \delta_{m}^{m}}{2^{m-2} M}\left(\frac{1+\delta}{\delta}\right)^{\bar{k}+1}\left(2+\frac{2}{\delta_{m}}\right)^{1+\bar{k}+m}\right)^{\frac{1}{p-1}-\frac{\mu}{2}-\bar{k}},
$$

which is positive. As by-product, the inequality (34) gives the lifespan estimate (12) and concludes the proof of Theorem 2.

\subsection{Proof of Theorem 1}

We start rewriting the Cauchy problem (1) as a nonlinear wave equation with a time-dependent potential. Let $v=v(t, x)$ be a solution of (1); we define

$$
u(t, x):=(1+t)^{\frac{\mu}{2}} v(t, x) .
$$

Then, the function $u=u(t, x)$ is a solution of the Cauchy problem

$$
\left\{\begin{array}{l}
u_{t t}-\Delta u=(1+t)^{-\frac{\mu}{2}(p-1)}|u|^{p}+\left(\frac{\mu}{2}\left(\frac{\mu}{2}-1\right)-v\right) \frac{u}{(1+t)^{2}}, \quad t \geq 0, \quad x \in \mathbb{R}^{n}, \\
u(0, x)=0, \\
u_{t}(0, x)=\varepsilon g(x) .
\end{array}\right.
$$


If $g$ is radial, then $u$ is radial and it satisfies Eqs. (13) and (14) with

$$
F(t, u)=(1+t)^{-\frac{\mu}{2}(p-1)}|u|^{p}+\left(\frac{\mu}{2}\left(\frac{\mu}{2}-1\right)-v\right) \frac{u}{(1+t)^{2}} .
$$

Let us fix $\delta>0$ and use the same notation of the proof of Theorem 2. Since we are assuming $\frac{\mu}{2}\left(\frac{\mu}{2}-1\right)-v \geq 0$, by comparison lemma, see [15, Lemma 2.9], we deduce $u>0$ in $\Sigma_{\delta}$. Then, it holds

$$
F(t, u) \geq(1+t)^{-\frac{\mu}{2}(p-1)}|u|^{p}
$$

hence, by formula (15) in Lemma 1 we still derive the estimate (20). Thus, the proof of Theorem 2 guarantees the result of Theorem 1.

Funding Open access funding provided by Politecnico di Torino within the CRUICARE Agreement.

Open Access. This article is licensed under a Creative Commons Attribution 4.0 International License, which permits use, sharing, adaptation, distribution and reproduction in any medium or format, as long as you give appropriate credit to the original author(s) and the source, provide a link to the Creative Commons licence, and indicate if changes were made. The images or other third party material in this article are included in the article's Creative Commons licence, unless indicated otherwise in a credit line to the material. If material is not included in the article's Creative Commons licence and your intended use is not permitted by statutory regulation or exceeds the permitted use, you will need to obtain permission directly from the copyright holder. To view a copy of this licence, visit http://creativecommons.org/licenses/ by/4.0/.

Publisher's Note Springer Nature remains neutral with regard to jurisdictional claims in published maps and institutional affiliations.

\section{REFERENCES}

[1] M. D'Abbicco. The threshold of effective damping for semilinear wave equations. Mathematical Methods in the Applied Sciences 38 (2015), 1032-1045.

[2] M. D'Abbicco, S. Lucente. NLWE with a special scale invariant damping in odd space dimension. Dynamical systems, differential equations and applications. 10th AIMS Conference. Suppl. (2015), $312-319$.

[3] M. D'Abbicco, S. Lucente, M. Reissig. A shift in the Strauss exponent for semilinear wave equations with a not effective damping. Journal of Differential Equations 259 (2015), 5040 - 5073.

[4] G. Girardi, S. Lucente. Lifespan estimates for a special quasilinear time-dependent damped wave equation. Proceedings of the 12th ISAAC congress in Aveiro - Session Recent Progress in Evolution Equations. 2020. In press.

[5] V. Georgiev, H. Lindblad, C. D. Sogge. Weighted Strichartz estimates and global existence for semilinear wave equations. American Journal of Mathematics (1997), 1291-1319.

[6] M. Ikeda, T. Tanaka, K. Wakasa. Critical exponent for the wave equation with a time-dependent scale invariant damping and a cubic convolution. (2020), arXiv:2003.10329.

[7] H. Kubo, Slowly decaying solutions for semilinear wave equations in odd space dimensions. Nonlinear Analysis: Theory, Methods \& Applications. 28 (1997), 327-357.

[8] H. Kubo, K. Kubota. Asymptotic behaviors of radially symmetric solutions of $\square u=|u|^{p}$ for super critical values p in even space dimensions. Japanese journal of mathematics. New series 24 (1998), $191-256$. 
[9] W. Nunes do Nascimento, A. Palmieri, M. Reissig. Semi-linear wave models with power nonlinearity and scale-invariant time-dependent mass and dissipation. Math. Nachr. 290 (2017), 17791805.

[10] A. Palmieri. A global existence result for a semilinear scale-invariant wave equation in even dimension. Mathematical Methods in the Applied Sciences, 42 (2019) 2680-2706.

[11] A. Palmieri. Global Existence Results for a Semilinear Wave Equation with Scale-Invariant Damping and Mass in Odd Space Dimension, New Tools for Nonlinear PDEs and Application, (2019), 305-369.

[12] A. Palmieri, M. Reissig. A competition between Fujita and Strauss type exponents for blow-up of semi-linear wave equations with scale-invariant damping and mass, J. Differential Equations 266, (2019), 1176-1220.

[13] A. Palmieri, Z. Tu. Lifespan of semilinear wave equation with scale invariant dissipation and mass and sub-Strauss power nonlinearity, Journal of Mathematical Analysis and Applications 470(1), (2019), 447-469.

[14] T. C. Sideris. Global behavior of solutions to nonlinear wave equations in three dimensions. Communications in Partial Differential Equations 8 (1983), 1291-1323.

[15] H. Takamura. Blow-up for semilinear wave equations with slowly decaying data in high dimensions. Differential Integral Equations 8 (1995), 647-661.

\section{Felisia Angela Chiarello}

Department of Mathematical Sciences "G. L. Lagrange”

Politecnico di Torino

Corso Duca degli Abruzzi 24

10129 Torino

Italy

E-mail: felisia.chiarello@polito.it

\section{Giovanni Girardi}

Department of Mathematics

University of Bari

Via Orabona $n .4$

Bari

Italy

E-mail: giovanni.girardi@uniba.it

Sandra Lucente

Department of Physics

University of Bari

Via Orabona $n .4$

Bari

Italy

E-mail: sandra.lucente@uniba.it

Accepted: 9 April 2021 